\title{
High-Performance Time-Code Diversity Scheme for Shore-to-Sea Maritime Visible-Light Communication
}

\author{
Hyeongji Kim, Atul Sewaiwar, and Yeon-Ho Chung* \\ Department of Information and Communications Engineering, Pukyong National University, \\ Busan 608-737, Korea
}

(Received May 4, 2015 : revised August 19, 2015 : accepted September 2, 2015)

\begin{abstract}
This paper presents a novel shore-to-sea maritime data transmission system based on time-code diversity, using visible light in maritime environments to overcome the limitations of conventional maritime wireless communications. The proposed system is primarily comprised of existing LED-based lighthouses and maritime transceivers (marine beacons, buoys, etc.), and thus is considered cost-effective in terms of implementation. We first analyze maritime visible-light communications on the basis of the unique properties of a maritime environment, i.e. sea states (wave height, wind speed, etc.), plus atmospheric turbulence, using the Pierson-Moskowitz (PM) and JONSWAP (JS) spectrum models. It is found that the JS model outperforms the PM model, and that the coverage distance depends on the LED power and sea states. To combat maritime fading conditions that significantly degrade performance and coverage distance, we propose a time-code diversity (TCD) scheme in which the delayed versions of the original data are retransmitted using orthogonal Walsh codes. This TCD scheme is found to be superior, in that it offers three orders of magnitude in terms of BER performance, compared to a conventional (non-TCD) transmission scheme. The proposed scheme is robust and efficient in overcoming the effect of impairments present in maritime environments with a BER of approximately $10^{-5}$ and a data rate of $100 \mathrm{Kbps}$ at a distance of $1 \mathrm{~km}$.
\end{abstract}

Keywords: Maritime communication, VLC, Time-code diversity, Pierson-Moskowitz model, JONSWAP model

OCIS codes : (200.2605) Free-space optical communication; (230.3670) Light-emitting diodes; (010.4450) Oceanic optics

\section{INTRODUCTION}

World trade depends significantly on ocean-based shipping. About 80 percent of foreign trade is conducted by marine transport [1]. An increase in maritime traffic has resulted in many large and small marine accidents each year, causing serious problems for human safety and marine environments. For a narrow shipping lane like Japan-Korea, which provides a vital passage to ships carrying goods traded between those nations, the safety of ships is crucial. To increase safety and security, e-Navigation or maritime communication is a concept developed under the auspices of the International Maritime Organization (IMO) for commercial shipping through better organization of data on ships and on shore, and better exchange and communication between the two
[2]. Conventional maritime wireless communications available at sea rely mainly on satellite links such as TRI-media Telematic Oceanographic Network [3] and European Space Agency [4], which are expensive and slower than HF and VHF communications. Radio-frequency (RF) maritime communication systems satisfy communication and navigation needs, at the expense of high cost and low transmission speed. Modern communications technology has provided high-speed transmission to users, but while terrestrial regions enjoy high data rates, marine environments are often limited by relatively low data rates from wireless communications for sea users (ship crews, etc.). For this reason, maritime communications need an advanced technology to provide high-speed transmission for sea users.

In recent years a new wireless technology known as visible-

\footnotetext{
*Corresponding author: yhchung@pknu.ac.kr

Color versions of one or more of the figures in this paper are available online.
} 
light communication (VLC) has come into the limelight. In VLC the blinking of a light-emitting diode (LED) is used for communication and illumination simultaneously [5]. LED communication offers innovative wireless technologies in terms of communication speed, flexibility, usability, and security. Unseen by the human eye, this blinking is used to carry high-speed data, creating a wireless communication network using existing light resources to achieve low-cost communication. In areas of maritime VLC, a few interesting reports have been documented. The authors reported simulation results using sea-state models for a shore-to-sea maritime VLC [6, 7], while underwater VLC was presented in [8]. Although introductory studies have been reported in the literature, a detailed and comprehensive study of VLC in maritime environments is yet to be documented in the literature.

In maritime environments, VLC can be applied to existing infrastructure such as lighthouses and maritime transceivers (marine beacons, buoys). Most of the lighthouses that use electric lamps have switched to LEDs now, because the life span, efficacy, and power consumption of an LED are far better than those of a conventional electric lamp. As part of a pioneering initiative, the Lighthouse Sub Project [9] was begun in September 2007, for the realization of long-distance VLC using an existing LED lighthouse. The project was able to record a transmission rate of $1200 \mathrm{bps}$ at a distance of $1 \mathrm{~km}$ and $1022 \mathrm{bps}$ at $2 \mathrm{~km}$ using imagesensor-based VLC. However, this project was conducted under silent sea conditions and did not include a study of the feasibility of maritime VLC under various sea states. Thus, further investigations under various ocean and atmospheric conditions are needed to establish a comprehensive maritime VLC system.

With this motivation to provide an efficient VLC link for maritime environments, it is important to analyze the effects of transmission under various atmospheric conditions and sea states (spectrum of sea waves due to blowing wind, atmosphere turbulence, etc.). We employ sea states drawn from the Pierson-Moskowitz (PM) and JONSWAP (JS) spectrum models under atmospheric turbulence conditions to evaluate the performance of the proposed scheme in maritime environments.

In this paper, we first present a comparative study of a maritime VLC link under atmospheric turbulence conditions using the PM and JS spectrum models, employing the On-Off Keying (OOK) scheme. Recognizing the performance degradation over distances larger than $1 \mathrm{~km}$, we propose a novel time-code diversity (TCD) scheme using the delayed versions of the original signal and orthogonal Walsh codes for maritime VLC environments. The proposed TCD scheme has the advantage of simplicity to achieve a reasonable diversity gain, resulting in a significant performance improvement compared to related schemes for maritime environments, such as adaptive optics and forward error correction. With the proposed scheme, the range of communication in maritime environments can be significantly improved, leading to a low-cost, high-speed wireless link in the shore-to-sea maritime VLC system.

The rest of this paper is organized as follows: Section II introduces the maritime VLC system together with the channel model. Performance analysis and simulation results are presented in Section III. Finally, Section IV shows the conclusions drawn from the investigation.

\section{SYSTEM CONFIGURATION}

\subsection{Maritime VLC System}

We first present a VLC-based maritime system that covers shore-to-sea communication services. Figure 1 shows the proposed system, in which the communication coverage area can be increased at a very low cost of implementation. The network is formed by neighboring ships, marine beacons, and buoys. Maritime transceivers are placed to connect to the terrestrial networks via VLC link. Lighthouses are assumed to operate as base stations. A base station consists of power LEDs that provide coverage to a very large area, while each maritime transceiver consists of an LED array and photodetectors.

\subsection{Maritime VLC Channel Modeling}

Maritime communication environments have a unique character, in which surface motion of the sea is usually observed and subsequently periodic degradation occurs. It is mainly characterized by this sea-surface movement, together with radio propagation and the Fresnel effect [10].

Sea waves are generated by the effect of wind passing over the ocean surface. Sea spectra are used for insight into the movement of the sea's surface in oceanography and ocean engineering. The waves are described only by a characteristic wave height (e.g. the significant wave height) and a characteristic period (e.g. the significant wave period or the peak period, i.e. the inverse of the peak frequency of the spectrum), or by a universal one- or two-dimensional spectrum [11]. The most accepted and renowned mathematical models for analysis of sea spectra are one-dimensional

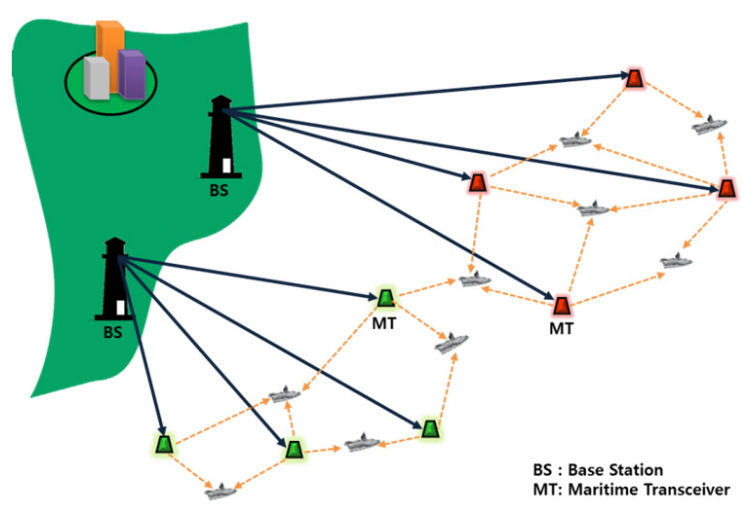

FIG. 1. Maritime VLC network. 
spectra: the Pierson-Moskowitz (PM) spectrum and the JONSWAP (JS) spectrum.

The PM spectrum is given by [11]

$$
E_{P M}(f)=\alpha_{P M} g^{2}(2 \pi)^{-4} f^{-5} \exp \left[-\frac{5}{4}\left(\frac{f}{f_{\text {peak }}}\right)^{-4}\right]
$$

where $\alpha_{P M}$ is the energy scale, $f$ is the wave frequency in $\mathrm{Hz}, g$ is the gravitational acceleration, and $f_{\text {peak }}$ is the peak frequency. The peak frequency depends only on the wind speed. The PM spectrum assumes that if the wind blew steadily for a long time over a large area, the sea waves would come into equilibrium with the wind.

On the other hand, the JS spectrum is based on nonlinear and wave-wave interactions for very long times and distances, from analysis of data collected during the Joint North Sea Wave Observation Project [12]. The JS spectrum is given by [11]

$$
E_{J S}(f)=\alpha_{J S} g^{2}(2 \pi)^{-4} f^{-5} \exp \left[-\frac{5}{4}\left(\frac{f}{f_{\text {peak }}}\right)^{-4}\right] \gamma^{\beta}
$$

where $\alpha_{J S}=0.076\left(\frac{U_{N}}{f_{\text {peak }} g}\right)^{0.22}, \quad \beta=\exp \left[-\frac{1}{2}\left(\frac{f / f_{\text {peak }}-1}{\sigma}\right)^{2}\right]$.

The energy scale parameter $\alpha_{J S}$, peak enhancement factor $\gamma$, and peak width parameter $\sigma$ are called the shape parameters; as they change, the sea-state spectrum changes. When $f \leq f_{\text {peak }}, \sigma$ is equal to the left peak width, denoted by $\sigma_{a}$, and when $f>f_{\text {peak }}, \sigma$ is identical to the right peak width, denoted by $\sigma_{b}$. Furthermore, $U_{N}$ is the wind speed measured by anemometer installed on a weather ship at a height of $N \mathrm{~m}$ above the sea surface. Since the JONSWAP model has a sharper peak than the PM spectrum, a peak-enhancement function $\gamma^{\beta}$ is added to enhance its peak.

In addition, the JS spectrum model has been shown to be universally applicable for idealized fetch-limited conditions and also arbitrary wind conditions in deep water, including storms and hurricanes [11]. This experimental model is known to be more realistic than the PM spectrum model.

The sea's surface moves all the time, rendering link quality unstable. Wave movement continuously changes both orientation and height of a marine beacon, thus changing the beacon's gain and received signal power. Figure 2(a) shows the maritime channel model under sea states. It consists of a base station and maritime transceivers (beacons) placed over the sea.

Figure 2(b) shows the maritime channel model under a given sea state with atmospheric turbulence. Atmospheric turbulence also degrades the performance of a maritime

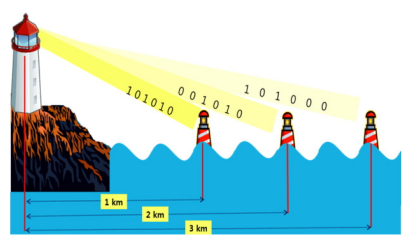

(a)

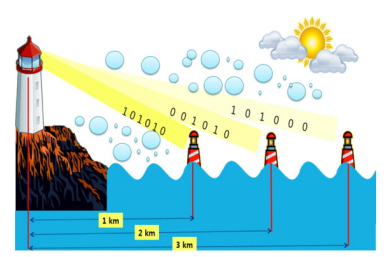

(b)
FIG. 2. Maritime channel model: (a) sea state, (b) sea state plus atmospheric turbulence.

TABLE 1. Sea-state parameters

\begin{tabular}{c|c|c|c|c}
\hline \hline \multirow{2}{*}{ Sea state } & Wind speed & $\begin{array}{c}\text { Average } \\
\text { wave period }\end{array}$ & $\begin{array}{c}\text { Significant } \\
\text { wave height }\end{array}$ & $\begin{array}{c}\text { Average } \\
\text { wavelength }\end{array}$ \\
\cline { 2 - 5 } & $(\mathrm{m} / \mathrm{s})$ & $(\mathrm{s})$ & $(\mathrm{m})$ & $(\mathrm{m})$ \\
\hline 4 & 9.26 & 5 & 1.83 & 24.1 \\
\hline 6 & 13.89 & 7.5 & 4.3 & 56.1 \\
\hline 8 & 22.5 & 13 & 13.72 & 180.1 \\
\hline
\end{tabular}

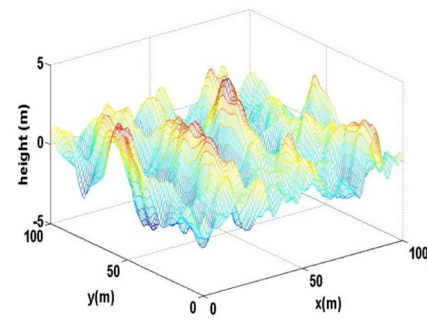

(a)

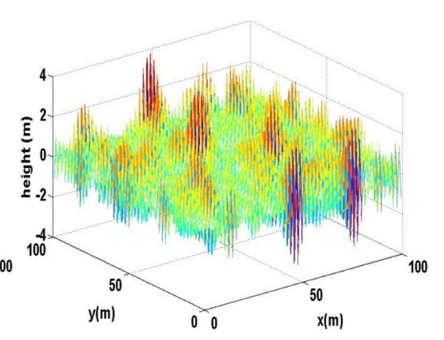

(b)
FIG. 3. Sea surface from the spectra under sea state 6: (a) PM (b) JS.

VLC link due to signal fading.

Since real-time data are not available for our study, we employ sea-state data generated by the PM and JS spectrum models described earlier. Table 1 shows the sea-state parameters [13]. The sea-state conditions mentioned in Table 1 are the result of the reflective nature of the sea surface and wave height, which are measures of the roughness of the sea. Figure 3 shows the exemplary sea surface of sea state 6 for PM and JS spectra.

For lower-numbered sea states (i.e. states 1 to 4 ) the sea conditions are mild, and hence the signal distortion is negligible at the receiver, while for the higher states conditions are hostile, with relatively high waves and fast winds. Such conditions scatter incoming signals, causing them to be extremely impaired at the receiver [14].

Atmospheric turbulence causes discrete cells or eddies of different temperatures that act like prisms of different sizes and refractive indices. Consequently, it degrades the performance of a VLC link. These regimes are also a function of the distance travelled by the optical radiation through the atmosphere. Atmospheric turbulence is usually 
categorized into regimes depending on the magnitude of variation and inhomogeneity of the refractive index. The log-normal distribution is generally used to model signal fading associated with the regime of weak atmospheric turbulence [15]. In the following analysis, weak turbulence is assumed, and thus the log-normal atmospheric model is used. The probability density function of the log-normal distribution for the received irradiance $I$ is given by [15]

$$
P(I)=\frac{1}{\sqrt{2 \pi \sigma_{l}^{2}}} \frac{1}{I} \exp \left\{-\frac{\left(\ln \left(I / I_{o}\right)+\sigma_{l}^{2} / 2\right)^{2}}{2 \sigma_{l}^{2}}\right\} \quad \mathrm{I} \geq 0,
$$

It is characterized by the log-irradiance variance $\sigma_{l}^{2}$, a mean value of $\sigma_{l}^{2} / 2$, and signal irradiance without scintillation $I_{0}$.

\subsection{Time-code Diversity}

To improve the performance of maritime VLC under various sea states, we propose time-code diversity (TCD), in which the original signal and its delayed versions are transmitted and then orthogonal Walsh codes of length 4 are employed. The value of the delay $\tau$ can be decided based on the maximum coverage distance $d$. In this case the value of $\tau$ is calculated to be $10 \mu$ s, i.e. $\tau=d / c$, where $d=3 \mathrm{~km}$ and $c=3 \times 10^{8} \mathrm{~m} / \mathrm{s}$. In other words, a delay of 1 bit is assured, since the bit duration and the value of $\tau$ are identical at $10 \mu \mathrm{s}$. This transmission strategy would yield diversity gain through the reception of multiple transmitted signals. The block diagram of the transmitter for the proposed TCD-based VLC system is shown in Fig. 4(a). The data and their three delayed versions are spread using orthogonal codes. After spreading, the composite signal is formed by adding all the signals from the direct path and their delayed versions. This composite signal is transmitted by LEDs after multiplying by the frequency $f_{c}$. Then the signal propagates through the channel and suffers from various sea states and atmospheric turbulence conditions, as noted earlier. The transmitted signal is given by

$$
m^{\prime}(t)=\sum_{i=1}^{4} d(t-(i-1) \tau) c_{i} \cos 2 \pi f_{c} t
$$

where $d(\cdot) \in[0,1]$ and $\tau$ represents the path delay shown in Fig. 4(a). $c_{i}$ represents a code sequence separated by the code for $i \in[1,2,3,4]$.

At the receiver, an optical filter is installed before the photodetector to reduce the effect of ambient light noise and sunlight [16]. A block diagram of the receiver is shown in Fig. 4(b). The received signal is then passed through coherent demodulator to recover the low-pass signal. The filtered signal from the demodulator is then fed to extract the original data by multiplying with the respective orthogonal codes. The decision circuit then estimates the data. In this

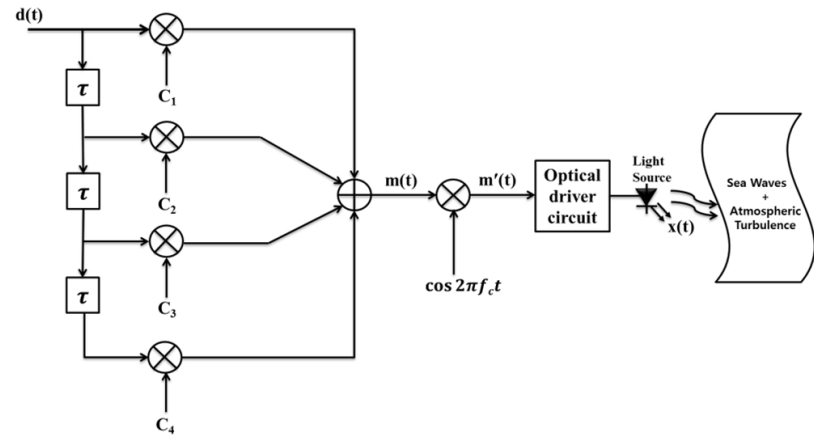

(a)

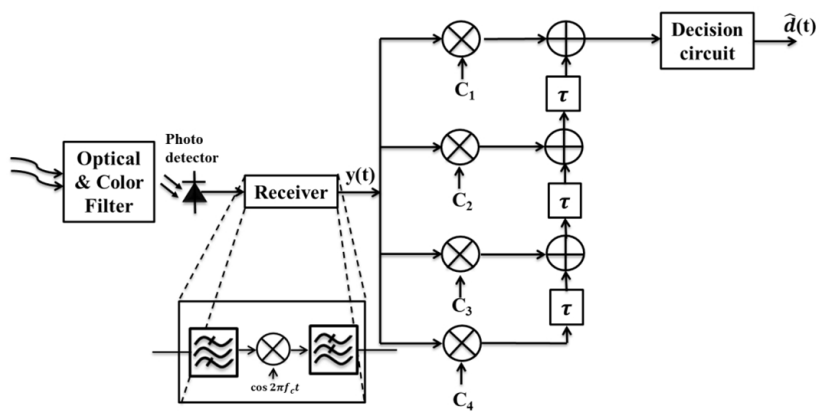

(b)

FIG. 4. Proposed time-code diversity scheme: (a) transmitter (b) receiver.

way the demodulated and estimated data are compared to those originally transmitted, to determine the bit error rate (BER).

The received signal can be modeled as [17]:

$$
y(t)=R I[1+\zeta m(t)]+n(t)
$$

where $R$ is the photodetector's responsivity, $I$ is the received optical irradiance, $\zeta$ is the modulation index, and $n(\mathrm{t})$ is additive white Gaussian noise with variance $\sigma^{2}$, computed from $\sigma^{2}=\left(\sigma_{B}\right)^{2}+\left(\sigma_{T}\right)^{2} . \sigma_{B}^{2}$ is the background noise, composed of shot noise due to radiation from sky and sun, and $\sigma_{T}^{2}$ is the total thermal noise at the receiver.

The sum of the demodulator outputs is fed into the decision circuit as shown in Fig. 4(b). At this point, the electrical signal-to-noise ratio (SNR) can be described as

$$
S N R=\frac{P_{m}}{\sigma^{2}}
$$

The average received optical power $P_{m}$ is given by

$$
P_{m}=\frac{A^{2}}{2 T} \int_{0}^{T} g^{2}(t) d t
$$

where $A$ is the symbol amplitude, $T$ is the symbol duration, 
and $g(t)$ is the pulse-shaping function that is included in the optical driver unit.

\section{SIMULATION RESULTS}

Simulations have been carried out to investigate the link performance for the proposed shore-to-sea TCD-based VLC system. Performance is evaluated in terms of BER versus SNR, with respect to the distance between base station and maritime transceivers. We used the parameters shown in Table 2 and employed the OOK modulation to model the maritime system. It is worth noting that a photodetector of physical area $2.8 \times 2.8 \mathrm{~cm}^{2}$ is commercially available [18].

In the maritime VLC link channel, the light from the transmitter is directed toward the receiver, where we measure the SNR and BER. The receiving plane is assumed to be $10 \mathrm{~m} \times 10 \mathrm{~m}$ about a beacon (maritime transceiver), as shown in Fig. 5 [6]. Also, all subsequent analyses are conducted under the sea state 6 , in terms of BER and SNR.

Figures 6 and 7 show the performance of the maritime VLC channel model under sea state 6 , where transmitter and receiver are separated by $1 \mathrm{~km}$. The JS spectrum

TABLE 2. Simulation parameters

\begin{tabular}{|c|c|}
\hline Parameter & Value \\
\hline $\begin{array}{c}\text { Transmitted optical power (All } \\
\text { LEDs) }\end{array}$ & $500 \mathrm{~W}$ \\
\hline Type of LED & RGB LED \\
\hline Number of LEDs & 100 \\
\hline Luminous intensity (All LEDs) & $12,732 \mathrm{~cd}$ \\
\hline Type of Photodetector & Si PIN \\
\hline Photodetector physical area & $3 \times 3 \mathrm{~cm}^{2}$ \\
\hline Bit duration / bit rate & $10 \mu \mathrm{s} / 100 \mathrm{Kbps}$ \\
\hline Field of view & $50^{\circ}$ \\
\hline $\begin{array}{c}\text { Distance between lighthouse } \\
\text { and receiver (D) }\end{array}$ & {$[1,1.5,2,2.5,3] \mathrm{km}$} \\
\hline $\begin{array}{c}\text { Tilting Angle of Photodetector } \\
\text { relative to D }\end{array}$ & $\begin{array}{c}{\left[\tan ^{-1}(0.05), \tan ^{-1}(0.033),\right.} \\
\tan ^{-1}(0.025), \tan ^{-1}(0.02) \\
\left.\tan ^{-1}(0.0167)\right]\end{array}$ \\
\hline $\begin{array}{l}\text { Refractive index of a lens at } \\
\text { receiver }\end{array}$ & 1.5 \\
\hline LED half angle & $60^{\circ}$ \\
\hline Delay $(\tau)$ & $10 \mu \mathrm{s}$ \\
\hline Frequency $(f)$ & $10 \mathrm{MHz}$ \\
\hline Receiving plane dimension & $10 \mathrm{~m} \times 10 \mathrm{~m}$ \\
\hline Channel & Maritime VLC Channel \\
\hline Height of the lighthouse & $50 \mathrm{~m}$ (above sea surface) \\
\hline $\begin{array}{c}\text { Height of the light source in } \\
\text { lighthouse }\end{array}$ & $48 \mathrm{~m}$ (above sea surface) \\
\hline
\end{tabular}

TABLE 3. Parameters of the maritime VLC channel

\begin{tabular}{c|c}
\hline \hline Parameter & Value \\
\hline$\alpha_{P M}$ & 0.0081 \\
\hline$\gamma$ & 3.3 \\
\hline$\sigma_{a}$ & 0.07 \\
\hline$\sigma_{b}$ & 0.09 \\
\hline$U_{N}$ & {$[9.26,13.89,22.5] \mathrm{m} / \mathrm{s}$} \\
\hline$I_{o}$ & 0.1 \\
\hline$\sigma_{l}^{2}$ &
\end{tabular}

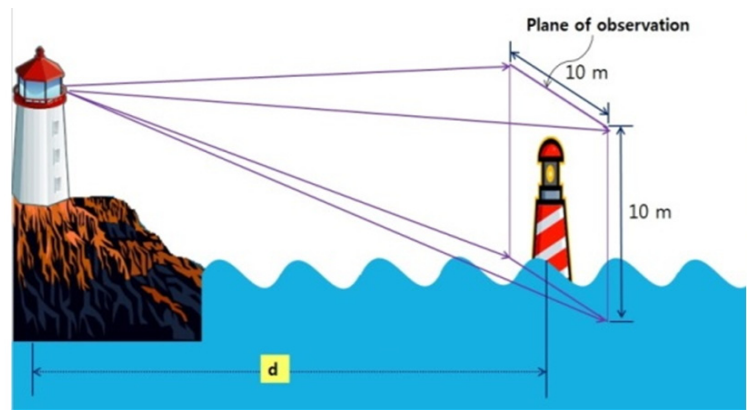

FIG. 5. Receiving plane $(10 \mathrm{~m} \times 10 \mathrm{~m})$.

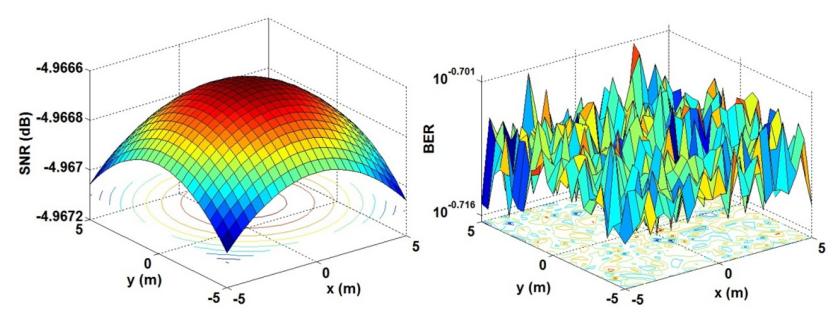

(a)

(b)

FIG. 6. Simulated maritime channel (PM, 1 km): (a) SNR (b) BER.

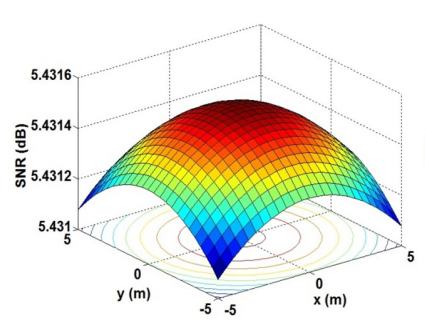

(a)

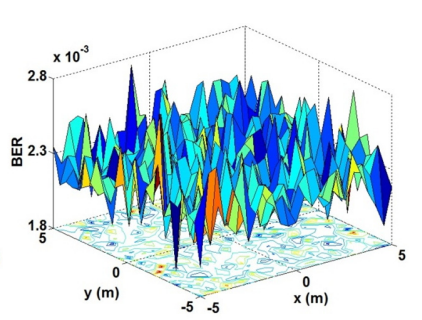

(b)
FIG. 7. Simulated maritime channel (JS, $1 \mathrm{~km}$ ): (a) SNR (b) BER.

model for sea state 6 shows a BER performance of $10^{-3}$ at a distance of approximately $1 \mathrm{~km}$, while the PM spectrum model for sea state 6 achieves a BER performance of approximately $10^{-0.70}$ at the same distance. Thus it can be concluded that the JS model, which is derived from empirical 
measurements, appears to be more realistic than the PM model.

Figures 8 and 9 show the analysis of the maritime VLC system under sea states 4,6 , and 8 using the OOK scheme. Overall the JS model outperforms the PM in terms of $\mathrm{BER}$, which is in agreement with the results mentioned in [7]. However, the BER performances at a larger distance between the transmitter and the receiver are poor, thus requiring higher LED power and a line-of-sight (LOS) path.

When atmospheric turbulence is included, the performance is slightly degraded, as shown in Fig. 9. We also perform simulations for various apertures of the photodetector. Observing the results from Fig. 10, it can be said that by increasing the aperture of the photodetector, the effect of atmospheric turbulence can be reduced.

Finally, we perform a comparative study for a representative transmission VLC scheme, i.e. conventional OOK, and the proposed TCD scheme. We also used the previously defined maritime VLC channel that is the JS model with atmospheric turbulence. The results of this comparative analysis are shown in Fig. 11. It is remarkable that the proposed scheme based on TCD yields a BER of approximately $10^{-5}$ at a

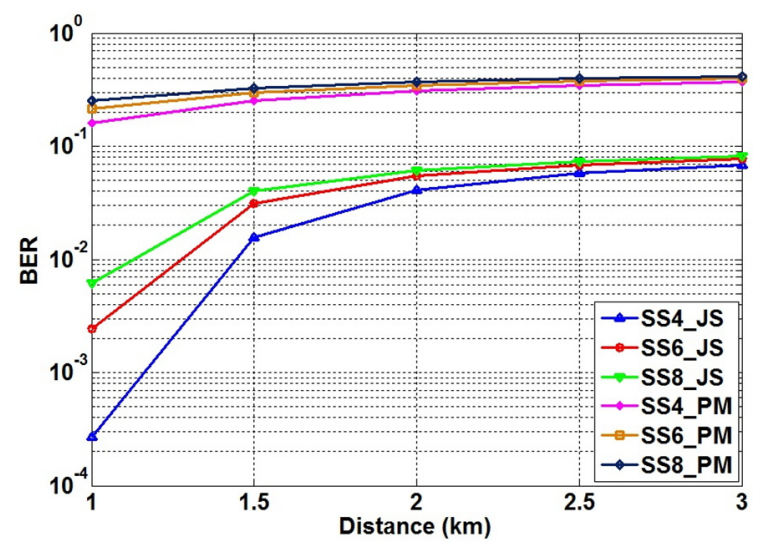

FIG. 8. Performance analysis for various sea states from JS and PM models.

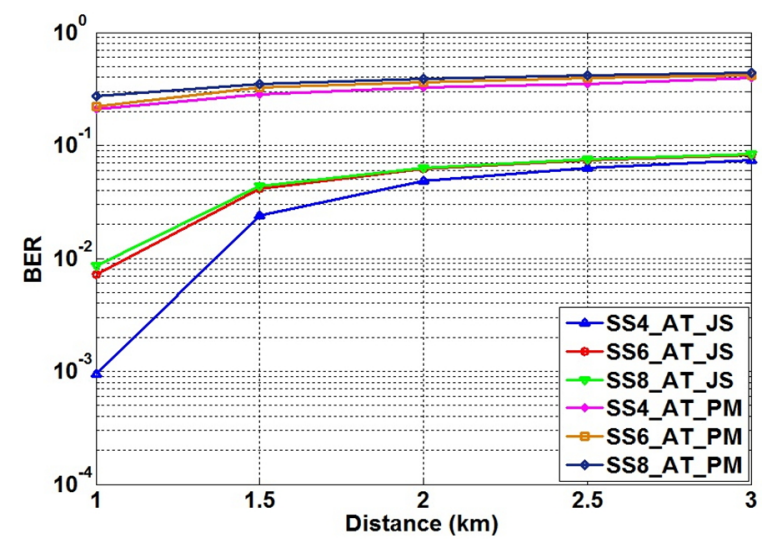

FIG. 9. Performance analysis for various sea states with atmospheric turbulence from JS and PM models.

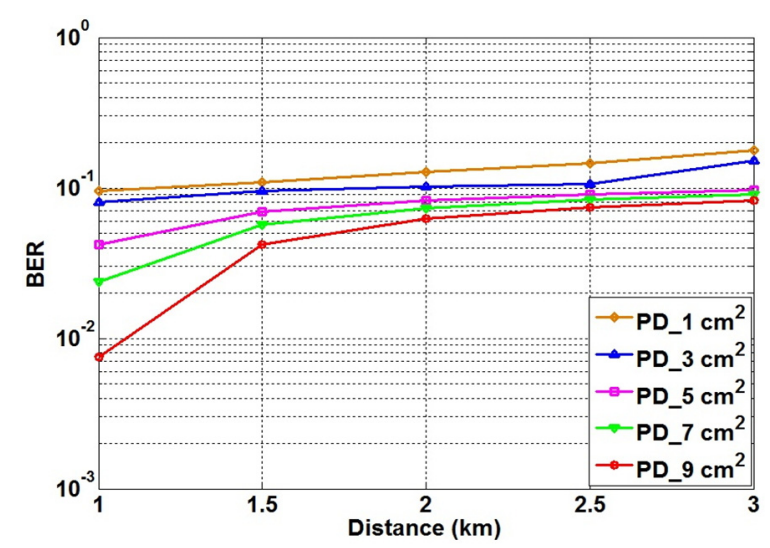

FIG. 10. BER performance for various physical areas of the photodetector (sea state 6 with atmospheric turbulence based on the JS model).

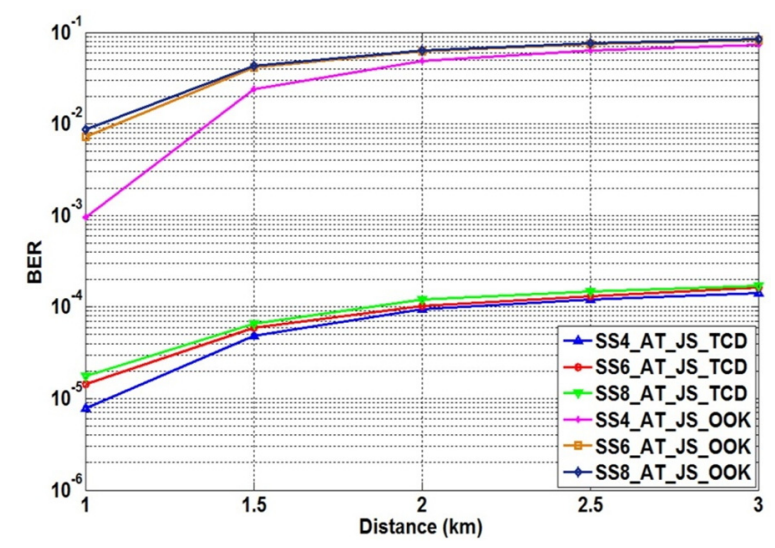

FIG. 11. Comparison of BER performance of OOK and TCD maritime VLC systems for sea states with atmospheric turbulence from the JS model.

distance of $1 \mathrm{~km}$. Even when the distance is increased, it still outperforms the conventional OOK scheme. Overall it is found that the TCD offers three orders of magnitude in terms of BER performance, compared to the OOK transmission scheme. Therefore, the proposed TCD can be regarded as an excellent performance-enhancing scheme for maritime VLC transmission scenarios.

\section{CONCLUSION}

A maritime VLC system with the TCD scheme has been proposed and analyzed based on the JS and PM sea spectra under atmospheric turbulence. The proposed TCD maritime system features visible-light transmission using LEDs and photodetectors for communication between shore and sea. Performance evaluation has been conducted using the PM and JS models under atmospheric turbulence. The performance relative to the sea state varies in terms of distance. It is found that the JS model appears to be more 
realistic and yields better performance over a larger distance. However, it is observed that for high sea states the performance degrades significantly, thus requiring a more rigorous transmission scheme. The high-performance TCD scheme is proposed in conjunction with maritime VLC transmission for improved signaling, even under high sea states and over a greater distance. Simulation results reveal that the proposed TCD scheme is superior to the conventional OOK scheme over all sea states considered. Therefore, the proposed TCD-based maritime VLC is an attractive candidate for an advanced maritime broadband communications system.

\section{ACKNOWLEDGMENT}

This work was supported by the Pukyong National University Research Abroad Fund in 2014 (C-D-2014-0718).

\section{REFERENCES}

1. B. Nora, J. Storgard, and J. Lappalainen, "The impact of ship crews on maritime safety," Publications of the Centre for Maritime Studies, University of Turku, A64 (2013).

2. IALA, "The IALA definition and vision for e-Navigation," E-NAV2-output, 11 (2007).

3. J. S. Pathmasuntharam, P. Y. Kong, M. T. Zhou, Y. Ge, H. Wang, C. W. Ang, W. Su, and H. Harada, "TRITON: high speed maritime mesh networks," in Proc. IEEE 19th International Symposium on Personal, Indoor and Mobile Radio Communications (Palais des Festivals Cannes, France, Sep. 2008), pp. 1-5.

4. ESA Telecommunication \& Integrated Applications, available at http://telecom.esa.int.

5. P. P. Han, A. Sewaiwar, S. V. Tiwari, and Y. H. Chung, "Color-clustered multiple-input multiple-output visible light communication,” J. Opt. Soc. Korea 19, 74-79 (2015).

6. H. J. Kim, A. Sewaiwar, and Y. H. Chung, "Shore-to-sea maritime communication with visible light transmission,"
Recent Advances in Electrical Engineering Series 39, 68-71 (2014).

7. H. J. Kim, A. Sewaiwar, and Y. H. Chung, "Maritime visible light communication with sea spectrum models," International Journal of Communications 9, 73-76 (2015).

8. G. Cossu, R. Corsini, A. M. Khalid, S. Balestrino, A. Coppelli, A. Caiti, and E. Ciaramella, "Experimental demonstration of high speed underwater visible light communications," in Proc. The 2013 2nd International Workshop on Optical Wireless Communications (Northumbria University, Newcastle upon Tyne, UK, Oct. 2013), pp. 11-15.

9. Lighthouse Sub Project VLCC, available at http://www.vlcc.net.

10. K. Lee and H. Park, "Modulations for visible light communications with dimming control," IEEE Photon. Technol. Lett. 23, 1136-1138 (2011).

11. L. H. Holthuijsen, Waves in Oceanic and Coastal Waters (Cambridge University Press, Cambridge, UK, 2007).

12. D. E. Hasselmann, M. Dunckel, and J. A. Ewing, "Directional wave spectra observed during JONSWAP 1973," Journal of Physical Oceanography 10, 1264-1280 (1980).

13. Sea States, available at http://www.ec.gc.ca/meteo-weather/ 279AC7ED-E09D-4E2A-A884-57321EA46B24/Met\%20101 \%20Chapter\%203\%20Eng.pdf.

14. K. D. Ward, C. J. Baker, and S. Watts, "Maritime surveillance radar. Part 1: Radar scattering from the ocean surface," IEE Proceedings F 137, 51-62 (1990).

15. R. L. Phillips and L. C. Andrews, "Universal statistical model for irradiance fluctuations in a turbulent medium," J. Opt. Soc. Am. 72, 864-870 (1982).

16. Y. H. Kim and Y. H. Chung, "Experimental outdoor visible light data communication system using differential decision threshold with optical and color filters," Opt. Eng. 54, 040501 (2015).

17. W. O. Popoola, Z. Ghassemlooy, J. I. H. Allen, E. Leitgeb, and S. Gao, "Free-space optical communication employing subcarrier modulation and spatial diversity in atmospheric turbulence channel," IET Optoelectronics 2, 16-23 (2008).

18. S3584 Series $\left(2.8 \times 2.8 \mathrm{~cm}^{2}\right)$ Datasheet, available at http://www. hamamatsu.com/eu/en/product/category/3100/4001/4103/S35 84-08/index.html. 